\title{
Um objeto de aprendizagem com ênfase no planejamento para o Moodle
}

\author{
Thiago Reis da Silva - UFRN - thiagoreis@ppgsc.ufrn.br \\ Rommel Wladimir de Lima - UERN - rommel.wladimir@uern.br \\ Carla Katarina de Monteiro Marques - UERN - carlakatarina@uern.br \\ Roberto Douglas da Costa - IFRN - douglas.costa@ifrn.edu.br \\ Marcos Vinicius de Andrade Lima, PPgCC-UERN/UFERSA, \\ marcos.engsoft@gmail.com \\ Karl Hansimuller Alelaf Ferreira, PPgCC-UERN/UFERSA - \\ karlhansimuller@gmail.com
}

\begin{abstract}
Resumo: A crescente expansão do uso de computadores nas escolas, nos mais diversos niveis escolares, vem incentivado professores e educadores a utilizar as novas tecnologias nos processos de ensino-aprendizagem, como, por exemplo, os Objetos de Aprendizagem. Portanto, este artigo tem como objetivo apresentar o Objeto de Aprendizagem - Mapa de Conteúdo, desenvolvido no Ambiente Virtual de Aprendizagem MOODLE e centrado no processo de ensino-aprendizagem, utilizando o padrão SCORM, com base em ferramentas situadas no processo de ensinoaprendizagem e fundamentadas em teorias pedagógicas consolidadas. Para isso, as ferramentas pedagógicas: Mapa de Conteúdos e Mapa de Dependências, cujo desenvolvimento é uma metodologia de planejamento para disciplinas, são pensadas como modelos para Objetos de Aprendizagem.
\end{abstract}

Palavras-chaves: Objetos de Aprendizagem, SCORM, Ensino-aprendizagem, MOODLE, Planejamento.

\section{A learning object with emphasis on planning for Moodle}

Abstract: The rapidly increasing use of computers in schools in various school levels, has encouraged teachers and educators to use new technologies in teaching and learning, for example, Learning Objects. Therefore, this article aims to present the Object of Learning - Content Map, developed in the Virtual Learning Environment MOODLE and centered teaching-learning process, using the SCORM standard, based on the tools located in the teaching-learning and statements based on pedagogical theories. For this, the pedagogical tools: Content Map and Dependency Map, which is developing a methodology for planning disciplines, are thought of as models for Learning Objects.

Keywords: Learning Objects, SCORM, Teaching-Learning, MOODLE, Planning.

\section{Introdução}

No cenário atual, caracterizado pela sociedade do conhecimento e da informação com avanços tecnológicos que perpassam os mais diferentes espaços formativos, torna-se 
fundamental o avanço de pesquisas buscando associar os benefícios, usos e possibilidades da tecnologia como suporte para a prática pedagógica e otimização do processo ensino-aprendizagem (LEITE et. al., 2011) nos Ambientes Virtuais de Aprendizagem (AVA) (DIAS et. al., 2009).

Nesse contexto, este artigo tem como objetivo apresentar o Objeto de Aprendizagem Mapa de Conteúdo (OBA-MC), cuja criação se encontra inserido dentro do processo de planejamento e desenvolvimento de um curso ou disciplina no AVA Modular ObjectOriented Dynamic Learning Environment (MOODLE), utilizando o padrão Sharable Content Object Reference Model (SCORM) (ADL, 2012).

Através da utilização das ferramentas pedagógicas Mapa de Conteúdos e Mapa de Dependências (LIMA; FIALHO, 2011), em conjunto com o padrão SCORM, o OBAMC insere o processo de planejamento da disciplina ou curso, com base em objetivos educacionais.

Face ao exposto, este artigo encontra-se organizado da seguinte forma. A Seção 2 explora os conceitos relacionados aos Objetos de Aprendizagem. Na Seção 3 são abordadas as Ferramentas Pedagógicas Mapa de Conteúdos e Mapa de Dependências. $\mathrm{Na}$ Seção 4 é apresentado o OBA-MC, os resultados e por fim na Seção 5 são descritas as Considerações Finais e os Trabalhos Futuros.

\section{Objetos de Aprendizagem}

Um Objeto de Aprendizagem (OA) é um recurso, suplementar ao processo de ensinoaprendizagem (TAROUCO; FABRE; TAMASIUNAS, 2003). Uma definição que aproxima OA da informática argumenta que estes objetos são recursos digitais que podem ser utilizados para dar suporte ao ensino, e que são construídos de forma a dividir o conteúdo em pequenos módulos reutilizáveis em diversos ambientes seguindo os princípios da orientação a objetos (WILEY, 2000). Nesta perspectiva, com a crescente utilização de OA para apoiar atividades de ensino-aprendizagem, tem sido desenvolvidas padrões no sentido de padronizar a especificação e a estrutura dos OA, permitindo sua reusabilidade (DUTRA; TAROUCO; PASSERINO, 2010).

Dentre os padrões, Dutra e Tarouco (2006), destacam que o SCORM é o mais centrado na autonomia e autoaprendizagem, e atualmente é o mais popular e com o maior número de ferramentas de suporte.

O foco do padrão SCORM é a autoaprendizagem, ou seja, o aluno interage exclusivamente com o material, sem interferência de outros atores no sequenciamento. A ordem do que será ensinado já vem estabelecida no próprio arquivo (VALHLDICK; RAABE, 2008). O SCORM incorpora diversas especificações de OA e, desta forma, torna-se compatível com diversos AVA. Conforme Hodgins e Conner (2001), o padrão SCORM é um dos melhores exemplos de integração de OA com padrões de AVA.

Utilizaremos o padrão SCORM como padrão para o desenvolvimento do OBA-MC, tanto por ser um padrão amplamente difundido na literatura, como também por ser composto de uma série de outros padrões. Com o SCORM, é possível utilizar, inclusive, os recursos providos pelo padrão Learning Object Metadata (LOM). Assim, é possível se beneficiar de diversos recursos oferecidos por cada um dos padrões, por isto foi adotado para ser utilizado no OBA-MC. 


\section{Ferramentas Pedagógicas}

As ferramentas pedagógicas Mapa de Conteúdos (MC) e Mapa de Dependências (MD) proposta por Lima (2009), são utilizadas para auxiliar o desenvolvimento do OBA-MC. De acordo com Silva et. al. (2012a), a ferramenta MC, apresentada em Lima e Fialho (2011), tem como objetivo fortalecer o processo de ensino-aprendizagem através do fornecimento de um conteúdo mais significativo (AUSUBEL, 1976) para professor e aluno. Baseado nos conceitos de Mapas Conceituais (NOVAK; CAÑAS, 2006), o MC fortalece o processo de aprendizagem do aluno através da visualização gráfica do conteúdo programático da disciplina ou curso. Para o professor, a ferramenta consiste de um conjunto de interações onde o mesmo responde às questões apresentadas no algoritmo da Figura 1. Ao final das interações o MC (Figura 2) é gerado.

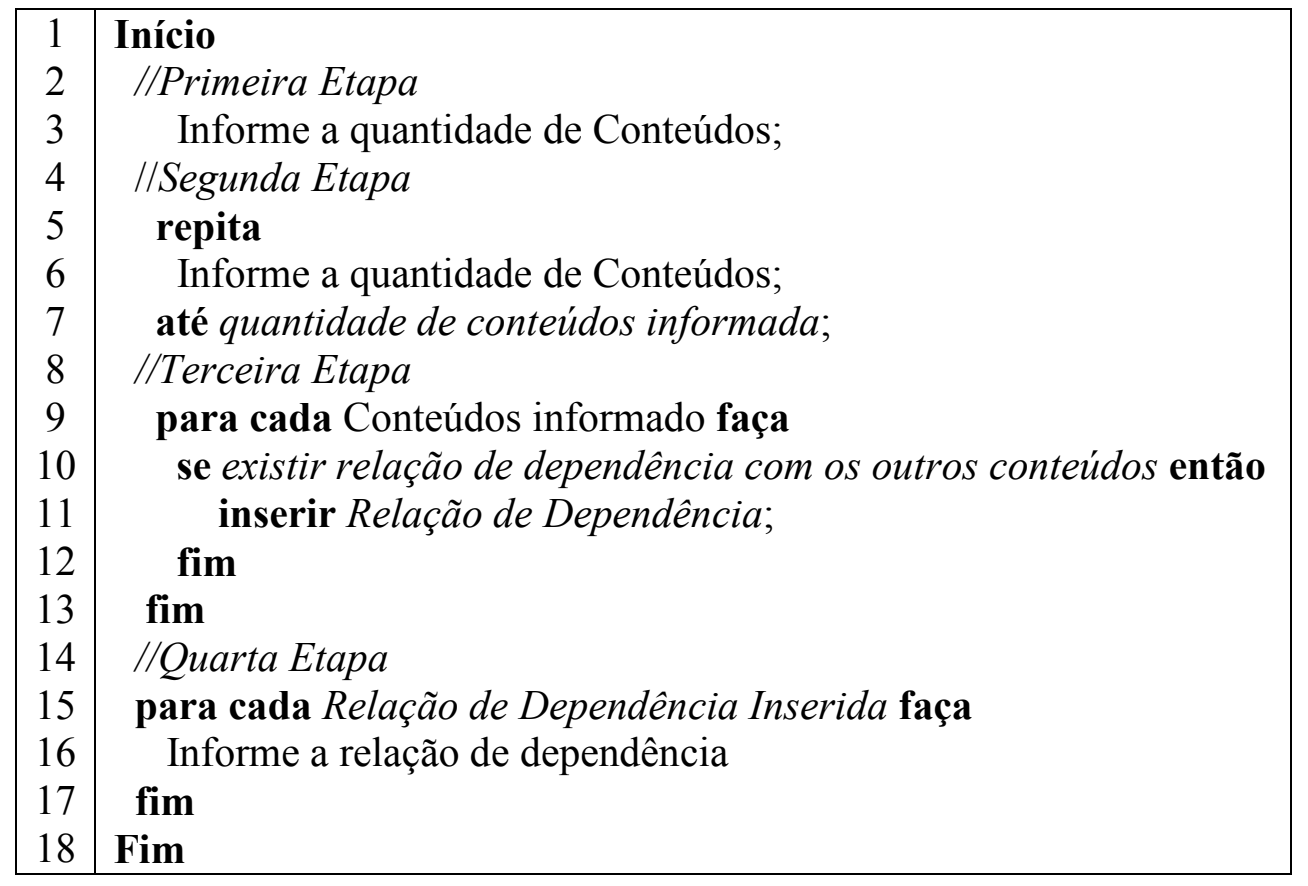

Figura 1 - Algoritmo de criação do MC.

Conforme Lima e Fialho (2011), o desenvolvimento do MC do curso é composto de quatro etapas (Figura 1). Na primeira etapa, o professor define a quantidade de conteúdos que existe para um determinado nível de visão da disciplina ou curso. $\mathrm{Na}$ segunda etapa o professor informa os nomes para identificação de cada um dos conteúdos, de acordo com a quantidade definida na fase anterior. Após a finalização dessa etapa, o MC da disciplina já possui os conteúdos que irão compor um determinado nível de visão. A partir desse momento tem início a terceira etapa da criação do MC da disciplina, através da definição das relações existentes entre os conteúdos identificados. Para cada um dos conteúdos deve ser investigado se o mesmo possui ou não dependência com os demais. Após a definição das relações existentes, tem início o desenvolvimento da quarta etapa, com a identificação dos nomes que serão utilizados para representar cada uma das relações apontadas na etapa anterior.

A ferramenta pedagógica MD é formada por um conjunto de Objetivos Educacionais $(\mathrm{OE})$, apresentados de forma gráfica, e relacionados entre si, através da Taxionomia de Bloom (BLOOM et. al. 1977). 


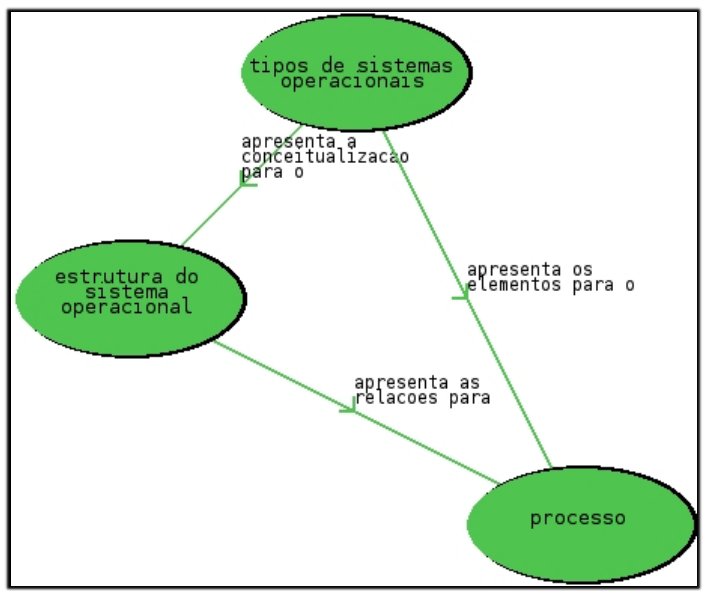

Figura 2 - Exemplo de um MC. Fonte: (SILVA et. al. 2012).

Como ilustra a Figura 3, o nível mais alto do MD representa o OE definido pelo professor para explicitar o que espera dos alunos em relação a um determinado conteúdo. Após esse primeiro nível, que pertence a uma determinada classe da taxionomia, o MD mostra a relação de dependência entre esse objetivo inicial e qualquer número de comportamentos da classe inferior que possam contribuir para a realização do objetivo inicial. Esse relacionamento de dependência se repete, com o MD podendo conter tantos níveis quantos forem necessários, até atingir a classe mais simples da Taxionomia de Bloom ou até que atinja um comportamento que não necessite de dependências (SILVA et. al. 2012a).

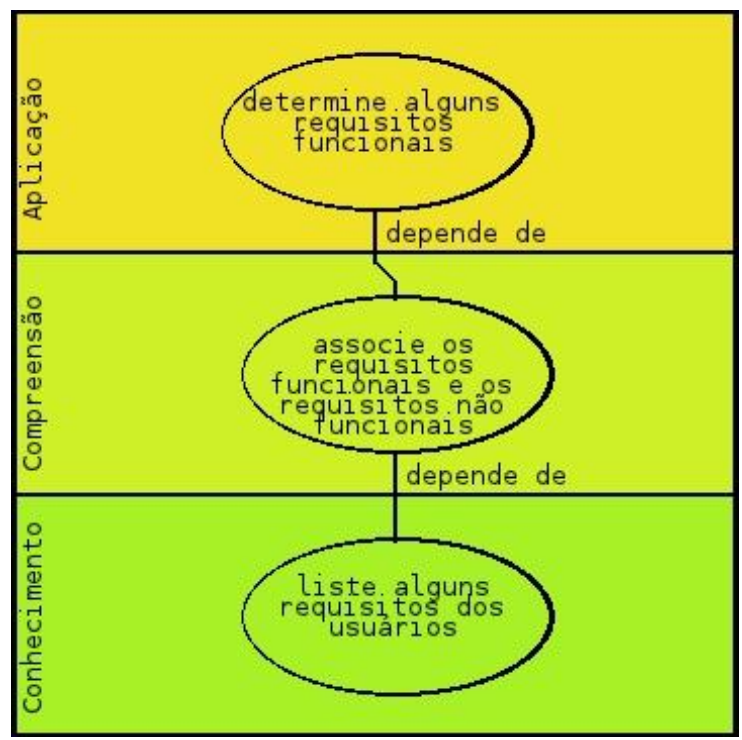

Figura 3 - Exemplo de um MD. Fonte: (SILVA et. al. 2012).

Neste contexto, o algoritmo da Figura 4 ilustra os passos para definição dos comportamentos esperados, para se atingir um objetivo em um determinado nível, e consequente o MD (Figura 3) é gerado.

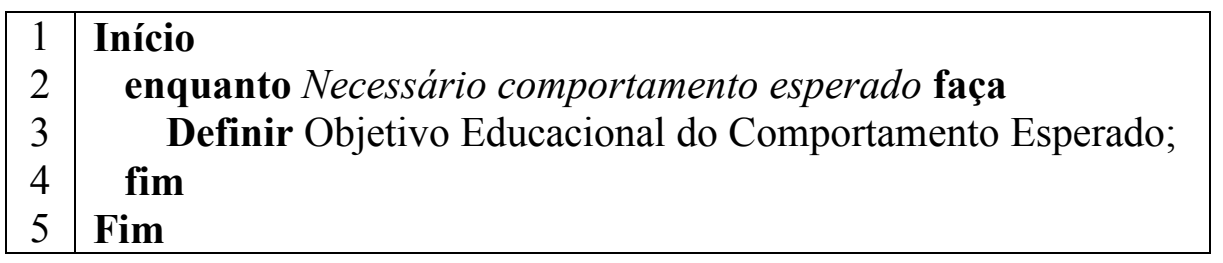

Figura 4 - Algoritmo de criação do MD. 
Por se tratar de ferramentas desenvolvidas com base em teorias pedagógicas consolidadas, o MC e o MD possibilitam o fortalecimento do processo e ensinoaprendizagem.

\section{OBA-MC}

Na criação de um OA é preciso pensar na sua reutilização. Assim sendo, para promover a reutilização de OA é necessária a padronização desses objetos para que possam funcionar corretamente em qualquer AVA. A padronização permite: uma fácil reutilização; a portabilidade dos conteúdos criados; a padronização dos processos de criação e a utilização em diferentes AVA (KRATZ et. al. 2007).

Sendo assim, o OBA-MC possibilita a criação de OA no AVA MOODLE, baseado no padrão SCORM, permitindo que os OA gerados possam ser utilizados em contextos educacionais diversificados.

O OBA-MC foi desenvolvido no AVA MOODLE, o qual tem como objetivo exportar os recursos educacionais criados a partir das ferramentas MC e MD. Para comprovar que as os recursos educacionais desenvolvidos por tais ferramentas são OA necessita-se firmar o conceito de reutilização dos OA, possibilitando sua execução em diferentes AVA. Para garantir a propriedade da reusabilidade dos OA, utilizou-se o padrão SCORM. Para auxiliar no processo de exportação do OA gerado foi desenvolvido um módulo, como ilustra a Figura 5.

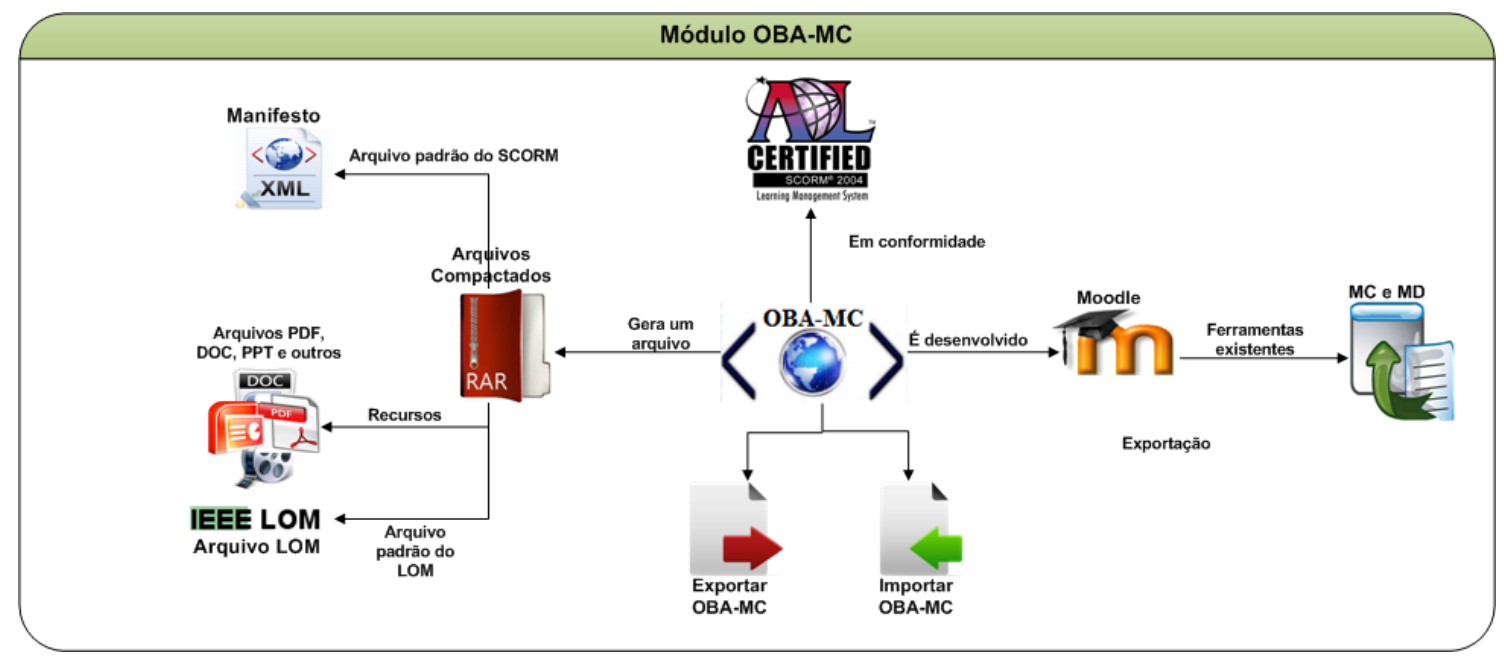

Figura 5 - Módulo OBA-MC.

Logo, este módulo tem como objetivo auxiliar nas duas etapas principais do OBA-MC, a etapa de importação e exportação do OA criado. Na criação do OBA-MC, é definidos os recursos que podem ser utilizados na criação de um uma aula, por exemplo, e como estes recursos podem ser agrupados em componentes maiores, formando, por exemplo, uma disciplina. Esses recursos são definidos de acordo com o SCORM em asset e Sharable Content Object (SCO). O asset é o recurso digital mais básico que pode ser utilizado dentro do conteúdo do OA, por exemplo, arquivos de texto, imagem ou outros elementos que tenham relação com o objeto criado. O SCO representa uma agregação de vários asset e tem o papel de fazer a comunicação com o AVA. A organização do conteúdo do OBA-MC, de acordo com a ferramentas MC e MD é dividida em dois grupos: atividades e recursos. Os recursos são formados por asset e SCO. As atividades são como módulos ou tópicos do OA, por exemplo, chat e fórum do AVA MOODLE. Cada OA criado pelo OBA-MC pode ser composto por várias atividades e/ou recursos. Após, todo esse processo de criação o OA é exportado. 
Na etapa de exportação, ocorre o processo de encapsulamento do OA em um pacote do tipo ZIP, garantindo a execução dos OA em outros AVA que tenha suporte ao SCORM. $\mathrm{O}$ pacote é composto por dois componentes principais. O primeiro é um arquivo eXtensible Markup Language (XML), conhecido como manifesto - imsmanifest.xml que descreve a estrutura e o conteúdo do OA e o segundo contém os arquivos que compõe o OA, tais como, arquivos .PDF, .PPT, páginas html, arquivos de áudio e vídeo, dentro outros.

A etapa de importação é a etapa externa ao processo de desenvolvimento do OBA-MC, aonde o criador do curso - em geral o professor - poderá ou não realizar a importação do modelo. Esta etapa será necessária quando o desenvolvedor do curso verificar possíveis adaptações do modelo criado.

Através do OBA-MC, o aluno será capaz de melhor perceber as relações entre os conceitos abordados em uma aula. Além disso, a interação do usuário com o OBA-MC resulta em gráficos, auxiliando a compreensão do fenômeno de forma qualitativa por parte do aprendiz.

\subsection{Resultados e Contribuições}

Como resultados iniciais, a Figura 6, ilustra os metadados criado pelo OBA-MC. Para isso, ele deve ser testado, com o propósito de certificar que seu pacote de conteúdo está correto e funcionando adequadamente. Para testar os metadados do OA criado, foi utilizada a ferramenta Reusable E-Learning Object Authoring and Delivery - Reload Editor (RELOAD, 2012). Nela é possível executar e reproduzir a funcionalidade do pacote de conteúdo SCORM, sem a necessidade de incluí-lo em AVA. Assim sendo, o OBA-MC pode ser distribuída em diferentes AVA, pois este modelo foi implementado sob um padrão presente e aceito por um grande número de AVA difundidos.



Figura 6 - Exibição dos Metadados produzidos pelo OBA-MC na ferramenta RELOAD. 
Como se observa, à esquerda da Figura 6 aparece à organização dos artefatos dentro do arquivo ZIP, à direita é detalhado o arquivo de manifesto. $\mathrm{O}$ destaque para o arquivo de manifesto ressalta a sua importância como estrutura complementar para a organização dos arquivos do OA. O formato ZIP não apenas reúne todos os artefatos em um único arquivo, como é capaz de reproduzir a estrutura hierárquica dos diretórios que compõem o objeto.

A Figura 7 ilustra um OA criado pelo OBA-MC com o auxílio das ferramentas MC e MD. Como podemos notar na imagem, existem algumas funcionalidades na ferramenta MC e MD, que tornará o OA criado amplo, como, por exemplo: Conteúdos (possibilidade de adicionar novos conteúdos ao OA), Recursos (inserção de arquivos e ou atividades como, por exemplo, arquivos PDF, DOC), Objetivos Educacionais (Taxionomia de Bloom) e outros.



Figura 7 - OA criado no MOODLE.

Como o OBA-MC foi desenvolvido utilizando o padrão SCORM, os resultados gerados podem ser executados em outros AVA, na Figura 8, pode-se observar a execução do objeto gerado no ATutor (ATUTOR, 2012). Destacamos que em outros AVA o OA desenvolvido não poderá ser editado, esta função só esta disponível para o MOODLE. Em outros AVA poderá além de visualização a inserção de outros recursos, tais como: arquivos de diversos formatos.

Desta forma, dentre outros resultados, podemos destacar algumas vantagens, que o OBA-MC, pode proporcionar ao processo de ensino-aprendizagem (SILVA et. al. 2011), tais como:

- Inserção de uma metodologia de planejamento com base em OE (metodologia de criação do MC e do MD);

- Utilização de teorias pedagógicas já consolidadas (Aprendizagem Significativa e Taxionomia de Bloom);

- Padronização do modelo de apresentação do OA (o objeto será apresentado de forma gráfica, seguindo os modelos do MC e MD); 
- Reutilização do OA (o modelo utilizado possibilita a integração entre objetos. Por exemplo, um determinado conteúdo em um MC pode fazer referência a outro OA).

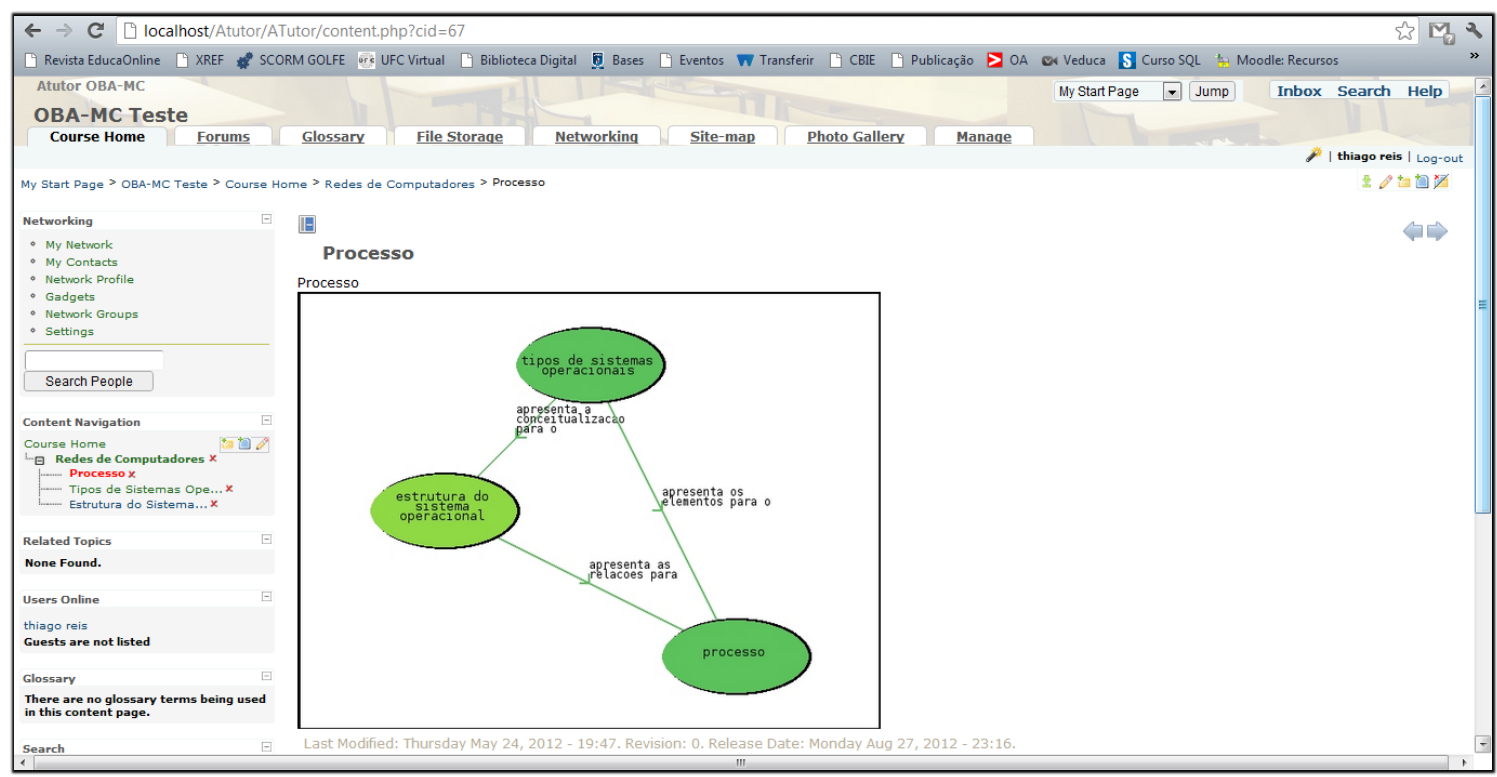

Figura 8 - Exibição do OA gerado pelo OBA-MC no AVA ATutor.

Além da portabilidade, que possibilita que os OA desenvolvidos pelo OBA-MC, possam ser executados pelos diversos AVA, este modelo proporcionará ao MOODLE algumas contribuições, tais como (SILVA et. al. 2012b):

- O planejamento das disciplinas e/ou cursos por meio de utilização do OBA-MC no MOODLE representa uma forma estruturada de desenvolvimento de material didático, pois durante esse processo os educadores são forçados a pensar em conteúdos menores, e não em toda a disciplina, para compor o curso e/ou a disciplina;

- A utilização de teorias pedagógicas destaca que o OBA-MC além de se preocupar com as características técnicas - utilização do padrão SCORM - o mesmo aborda as características pedagógicas, utilizando a Teoria da Aprendizagem Significativa e a Taxionomia de Bloom, auxiliado pelas ferramentas pedagógicas Mapa de Conteúdos e Mapa de Dependências;

- Os OA desenvolvidos pelo OBA-MC são conteúdos autocontidos, dessa forma podem ser utilizados em diversas situações, como, por exemplo, disciplinas distintas;

- $\mathrm{Na}$ construção do OBA-MC por partes dos professores, o mesmo terão metadados associados, que facilita a tarefa de busca e recuperação em ROA e também em AVA;

- Permite exportar e importar o OBA-MC e exibi-lo em outros AVA.

\section{Considerações Finais e Trabalhos Futuros}

Mesmo com a multiplicidade de definições para os OA, é possível perceber que a utilização das teorias pedagógicas da Aprendizagem Significativa e a Taxionomia de Bloom amplia a eficácia do uso de um OA visto que considera os processos cognitivos e a formação dos conceitos na cognição do aprendiz. 
A utilização do OBA-MC otimiza o desenvolvimento dos OA e padroniza seu formato bem como facilita o uso de outros objetos produzidos na mesma perspectiva de desenvolvimento.

Como trabalhos futuros pretende-se testar o modelo proposto em cenários educacionais mais realistas, como, a sua aplicação no planejamento de aulas por diferentes professores em diferentes áreas e também sua aplicabilidade em sala de aula.

\section{Agradecimentos}

Os autores agradecem a CAPES e a FAPERN pela concessão das bolsas de pesquisa e pelo apoio financeiro para realização da mesma.

\section{Referências}

ADL. Advanced Distributed Learning. 2012. Disponível em: <http://www.adlnet.org>. Acesso em mar. 2012.

AUSUBEL, D. P. Psicología educativa: un punto de vista cognoscitivo. Trad. Roberto Helier Domínguez. Trillas: México, 1976.

ATUTOR. Learning Content Management System. 2012. Disponível em: $<$ http://www.atutor.ca/>. Acesso em: mai. 2012.

CARVAlHO, L. A. S.; SANTOS, C. N. P.; ANDRADE, L. C.; CAVAlCANTE NETO, A. L. Planejamento: uma simples organização de conteúdo ou ferramenta pedagógica?. In: X Jornada de Ensino, Pesquisa e Extensão - JEPEX. Recife - PE. 2010.

BLOOM, B. S.; ENGELHART, M. D.; FURST, E. J.; HILL, W. H.; KRATHWOHL, D. R. Taxonomia de objetivos educacionais - domínio cognitivo. Globo: Porto Alegre - RS. 1977.

DIAS, C. L.; KEMCZINSKI, A. L.; SÁ, S. V.; FERLIN, J.; HOUNSELL, M. S. Padrões abertos: aplicabilidade em Objetos de Aprendizagem (OAs). In: XX Simpósio Brasileiro de Informática na Educação - SBIE. Florianópolis - SC. 2009.

DUTRA, R. L. S.; TAROUCO, L. M. R. Objetos de Aprendizagem: Uma comparação entre SCORM e IMS Learning Design. In: Revista Novas Tecnologias na Educação RENOTE, v.4, n.1, 2006.

DUTRA, R. L. S.; TAROUCO, L. M. R.; PASSERINO, L. Utilização de Objetos de aprendizagem abertos SCORM para dar suporte à avaliação formativa. In: Revista Brasileira de Informática na Educação - RBIE, v18, n3, p.59-69, 2010.

HODGINS, W.; CONNER, M. Everything you ever wanted to know about learning standards but were afraid to ask. 2001. Disponível em: $<$ http://linezine.com/2.1/features/wheyewtkls.htm>. Acesso: mai. 2012.

KRATZ, R. A.; PINTO, S. C. C. S.; SCOPEL, M.; BARBOSA, J. Fábrica de Adequação de Objetos de Aprendizagem. In: Revista Brasileira de Informática na Educação - RBIE. v. 15, n. 3, p. 25-38. 2007.

KLOSOUSKI, S. S.; REALI, K. M. Planejamento de ensino como ferramenta básica do processo ensino-aprendizagem. In: Revista Eletrônica Lato Sensu. Guarapuava: UNICENTRO, 5. ed. 2008.

LEITE, E. A. M.; SALES, G. L.; SOUSA, L. L. R.; JOYE, C. R. Avaliação Assistida, Feedbacks e Questionários do Moodle. In: Simpósio Brasileiro de Informática na 
Educação/XVII Workshop de Informática na Escola. p.2303-2313. 2011. Aracajú SE.

LIMA, R. W. Mapa de Conteúdos e Mapa de Dependências: ferramentas pedagógicas para uma metodologia de planejamento baseada em objetivos educacionais e sua implementação em um ambiente virtual de aprendizagem. Tese (Doutorado), UFRN, 2009.

LIMA, R. W.; FIALHO, S. V. Mapa de Conteúdos e Mapa de Dependências: ferramentas para um planejamento com base em objetivos educacionais. In: Revista de Exatas e Tecnológica - RETEC, v.2, p.10, 2011.

NOVAK, J. D.; CAÑAS, A. J. The theory undelying concept maps and how to construct them. Techinical Report IHCM CmapTools 2006-1, Florida Institute for Human and Machine Cognition, 2006.

RELOAD. Reusable eLearning Object Authoring and Delivery. 2012. Disponível em: $<$ http://www.reload.ac.uk>. Acesso em: out. 2012.

SILVA, T. R.; LIMA, R. W.; COSTA, R. D.; MARQUES, C. K. M. Uma proposta de padronização de Objetos de Aprendizagem com base em Objetivos Educacionais. In: Escola Potiguar de Computação e suas Aplicações - EPOCA. Natal - RN. 2011.

SILVA, T. R.; LIMA, R. W.; MESQUITA, H. H. O.; MARQUES, C. K. M.; COSTA, R. D.; ROCHA, M. P. T. Um modelo de Objetos de Aprendizagem centrando no processo de ensino/aprendizagem. In: VII Conferência Latino-americana de Objetos e Tecnologia de Aprendizagem - LACLO. Guayaquil, Equador, 2012.

SILVA, T. R.; LIMA, R. W.; MESQUITA, H. H. O.; MARQUES, C. K. M. O uso de ferramentas pedagógicas no desenvolvimento de Objetos de Aprendizagem. In: XXXII Congresso da Sociedade Brasileira de Computação - CSBC/I Workshop de Desafios da Computação Aplicada à Educação - DESAFIE. Curitiba - PR. 2012.

SILVA, T. R.; LIMA, W. R.; MESQUITA, H. H. O.; COSTA, R. D. Integrando um modelo de Objeto de Aprendizagem centrado no processo de ensino-aprendizagem a um curso do Moodle. In: VI MoodleMoot Brasil. São Paulo, SP, 2012.

TAROUCO, L. M. R.; FABRE, M.-C. J. M.; TAMUSIUNAS, F. R. Reusabilidade de objetos educacionais. In: Novas Tecnologias na Educação - RENOTE. v.1, n.1, fev. 2003.

VAHLDICK, A.; RAABE, A. L. A. Adaptação de Conteúdo SCORM em Ambientes Inteligentes de Aprendizagem. In: XIX Simpósio Brasileiro de Informática na Educação - SBIE. Fortaleza - CE. p. 52-61. 2008.

WILEY, D. A. Connecting learning objects to instructional design theory: a definition, a metaphor, and a taxonomy. 2000. Utah State University. Disponível em: $<$ http://reusability.org/read/chapters/wiley.doc>. Acesso em: jun. 2012. 